\title{
The Symmetries of the Icons on Ancient Seals
}

\begin{abstract}
Amelia Carolina Sparavigna ${ }^{1}$
${ }^{1}$ Department of Applied Science and Technology, Politecnico di Torino, Torino, Italy

Abstract: In this paper, we discuss the symmetries of the icons engraved on some ancient seals, in particular on the stamp seals. Used since the Neolithic times to impress some decorations, these early seals had probably the social function of allowing the identification of their owners too. The first seals were engraved with simple geometric images, then animals and humans appeared, arranged on their small circular or elliptic surfaces with bilateral and rotational symmetries.
\end{abstract}

Keywords: Symmetry, Seals, Scarab Seals

\section{Introduction}

We can find symmetries in human artifacts across time and cultures. As widely discussed, this is coming from a biological significance of the symmetry [1,2]. In fact, the simplest symmetry, the bilateral one, seems to be deeply related to a perception of health and beauty of a living being. Humans are very sensitive to the presence of symmetry in images [3] and many speculations about the mechanisms underlying the vision of symmetry have been proposed $[4,5]$.

The human sensitivity to symmetry, implicitly involved in the aesthetic sense of pleasure arising when good proportioned and balanced forms are observed [6,7], stimulates the use of symmetries in science too [8]. In physics, the symmetry is marking the existence of the crucial invariance of physical laws under specific transformations. Conservation of energy, momentum and angular momentum can be viewed as a consequence of the symmetry for continuous translations in time and space and for rotations, according to the Noether's Theorem [9]. In solid state physics, discrete symmetries govern the oscillations of the atomic structure and the mechanisms of charge and energy transport.

Studies on the symmetries in pottery decorations have been already performed [10]; relevant for the identification of a specific period of an ancient culture, symmetries have a practical use in the restoration of damaged materials too. In this paper, we will discuss the presence of symmetry in other ancient artefacts, the stamp seals. Considered as a form of art of non-primary importance, engraved images can be not only beautiful but also rather important for archaeological studies.

Ancient stamp seals and cylinder seals were made of a hard material and used to press an engraved figure into soft clay, for securing purposes [11], or, as in the case of for the early Neolithic seals, to stamp decorations on leather and textile. It is possible that they were used to stamp non-permanent tattoo, as guessed for seals found at Çatalhöyük (7500-5700 BCE) [12]. This use is guessed also for the pre-Columbian seals [13].

The seals were of two main types, cylinders and stamps. Cylinders firstly appeared in Mesopotamia in the late 4th millennium BCE, then widespread in Syria and Egypt and in the Aegean area. Stamp seals preceded cylinders. Early stamp seals were used in Persia, northern Syria, and Anatolia [11]. In Egypt, the scarab seals largely replaced cylinder seals early in the 2nd millennium BCE and continued to be the main type of used seals, until they were replaced by the signet rings during the Roman period.

For the Egyptians, a scarab seal was not only an impression seal: it was also an amulet with images and symbols engraved to protect the owner [14-18]. Stamp seals with round or elliptic shapes are considered as scarab seals, even when the round back of the object is not precisely shaped as a scarab. Egyptian scarabs have inscriptions with names of kings and gods and human and animals figures. In fact, many scarabs of the Middle Kingdom Period have the base decorated with coils and entanglements of cords. These decorations display bilateral symmetry (see Figure 1), sometimes with two- and four-fold rotational symmetry.

Seals collections are seldom published in the public domain, then it is impossible to be exhaustive in describing all the possible symmetries displayed by these objects. Therefore, let us use a different approach, that of following a time-line to see if any evolution in the symmetries of the decorations is present. 


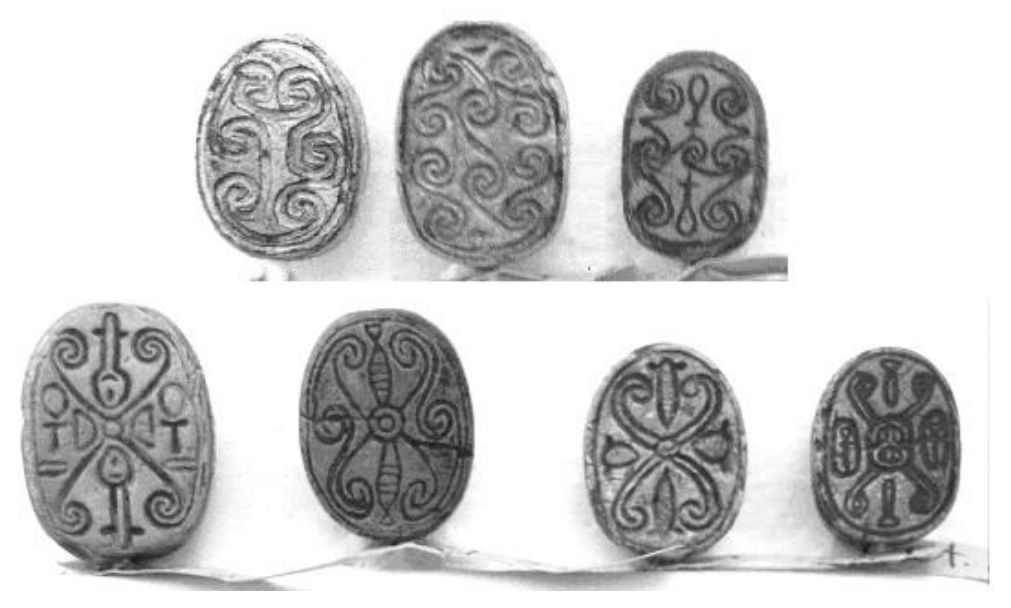

Figure 1. Seals of the Middle Kingdom with spiral coils and crosses, at the Egyptian Museum of Torino.

\section{Symmetries and seals}

Images on stamp seals can display those symmetries which are adequate to the shape of their bases. Mirror symmetry or bilateral, which occurs when two halves of a whole are each other's mirror images, and rotational symmetries with respect to rotations in the space can be observed. Rotations are about an axis perpendicular to the plane of the image: we find a twofold symmetry if we rotate the image of 180 degrees, a four-fold one when the rotation is of 90 degrees. Generally, we have $n$-fold rotation when angle is $2 \pi / n$.Because it is quite natural to follow the image in its rotation in a round space, the rotational symmetry produces a feeling of motion and evolution.
We can ask ourselves how old the rotational symmetry of decorative images is. Besides pottery, the other objects that can help us in investigating this question are the stamp seals. The drawing and pictures of Ref.12 can help us: they are showing the stamp seals found at Çatalhöyük (7500-5700 BCE), a very large Neolithic and Chalcolithic settlement in southern Anatolia. This is the largest and best preserved Neolithic site found to date. Images have geometric patterns with bilateral and rotational symmetries, usually a two-fold rotational symmetry (such as in the Figure 2). There is also a quite interesting stamp seal decorated with an icon that looks like a Ying-Yang symbol.

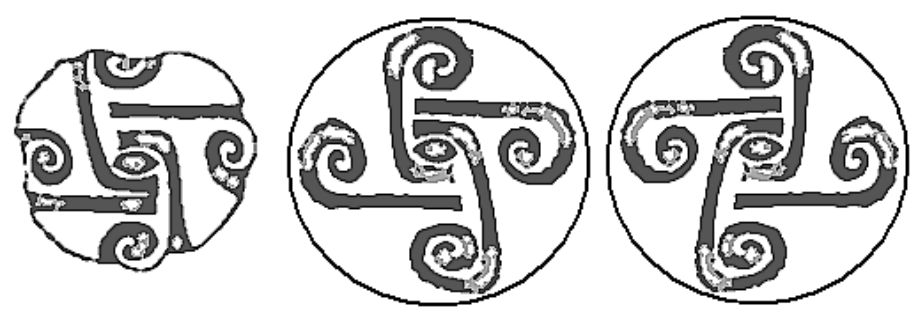

Figure 2. On the left, a seal from Çatalhöyük (redrawn from an image of Ref.12). The object seems to have its rim damaged. In the middle, we can guess a possible original icon on this stamp. On the right, the mirror image of its impression.

The impressing of carved stones into clay to seal containers had a long tradition in Near East, in particular in Mesopotamia, with the earliest evidence found in Syria dating to the seventh millennium BCE. During the Ubaid period, the variety of designs carved on seals expanded from the simple geometric forms to include animals with humans, snakes and birds. Seals decorated with four-legged horned animals can be easily found. In a stamp seal from the region North Syria - Iraq [19], dated 5th-4th millennium BCE, we see a standing male figure between two horned quadrupeds back to back and head to tail (see Figure 3a). The overall structure of the image is built to respect the two-fold rotational symmetry of the animals. On the right side of the Figure 3, we see a stamp seal from Susa, in Iran. Susa is one of the oldest known settlements of the world, probably founded about 4200 $\mathrm{BCE}$, although the first traces of an inhabited village have been dated to $7000 \mathrm{BCE}$. The seal depicts two goat-antelopes head to tail, and an oval at the center of the stamp [20]. In this seal, the idea of motion is strongly enhanced; the two antelopes seem to run on the rim of the seal. 

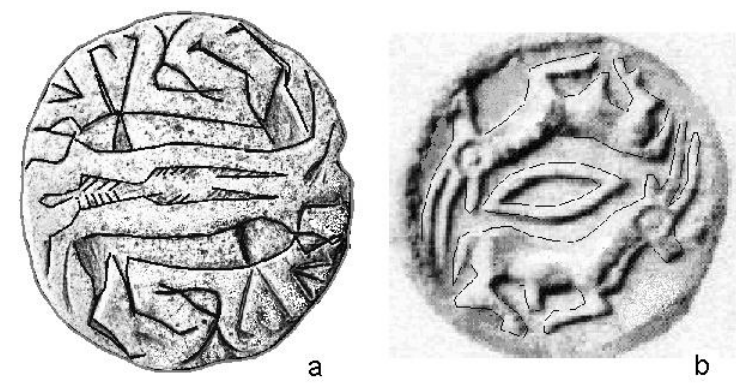

Figure 3. Images on a stamp seal from the region North Syria- Iraq (image redrawn from Ref.[19]), dated 5th-4th millennium BCE (a), and on a stamp from Susa (image redrawn from Ref.[20]), at the Louvre Museum (b).

In the same Ref.20, other seal images are showing bilateral symmetry, in particular the seals from the Dilmun culture. Dilmun was a land mentioned by Mesopotamian as a trade partner and as a trading post of the Mesopotamia and the Indus Valley Civilization trade route. It might be associated with the islands of
Bahrain. Figure 4 shows an example of a Dilmun seal, the left part of which has beautiful mirror symmetry, with stylistic influences from the Indus Valley. Stamp seals of the ancient Indus cities are also quite interesting, but they have a square shape.

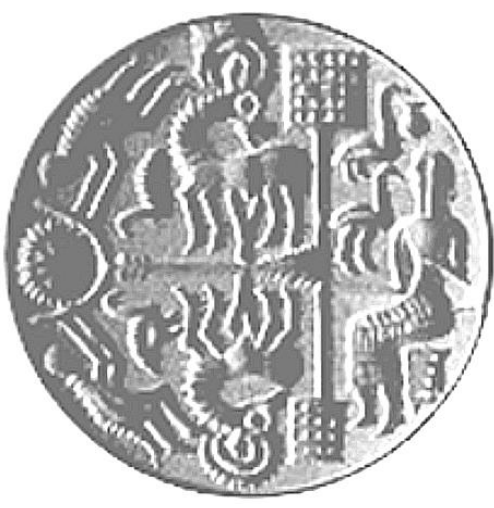

Figure 4. Stamp seal from Dilmun, redrawn from an image at http://www.uaeinteract.com

As told in the introduction, symmetry is a common phenomenon in human culture. This is easy to understand for bilateral symmetry which seems to be quite naturally associated with the fact that animals and human beings possess this symmetry. But other symmetries, which are rare in nature, for instance the two-fold rotational symmetry, are quite common in very old artefacts too. A possible reason for the appearance of rotational symmetries in decorations was proposed by P. Gendes. According to his researches $[21,22]$, these symmetries are naturally involved in the weaving activities. It is then not surprising that the earliest seals have geometric designs with rotational symmetries.

\section{Antisymmetries and broken symmetries.}

An antisymmetry in images means that we observe the mirror or the rotated image in inverted colours. When the image has a rotational anti-symmetry, we have an enhancement of the feeling of motion and evolution, due to the contrast of inter-penetrating colours. Let us consider for instance a well-know symbol, the YinYang symbol of Taoism. As in many religious symbols, the symmetry is used to convey an intuitive meaning. And in fact, we see that the ancient Taijitu image of Taoism has a fascinating use of symmetry for rotation about the central point (a two-fold one), combined with black-and-white inversion of colours (see Figure 5). The image actually intends to be a representation of the complementary need for male and female concepts. The rotational antisymmetry catches our attention with a feeling of evolution in a framework of complementary actions, not of mere recurrence. The following Figure 6 shows the use of bilateral antisymmetry to create interesting shadowing and three-dimensional effects. 


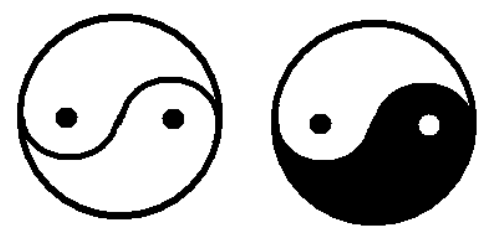

Figure 5. A two-fold rotation symmetry on the left gives an idea of recurrence. The anti-rotational symmetry is shown by the Yin-Yang symbol (on the right).

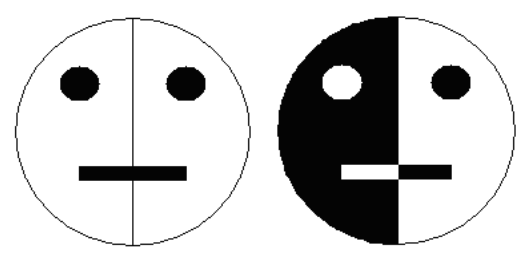

Figure 6. Bilateral antisymmetry can create a shadowing effect.

When seals are prepared to be used for stamp decorations on textiles, the creation of antisymmetric images with opposite colours is possible. In the case of those ancient seals, with images engraved in hard materials and used to stamp in clay, the creations of antisymmetries seems to be impossible. Nevertheless we can try to find an alternative meaning of antisymmetry for such seals. Let us consider the possibility illustrated in the Figure 7, on the left, which shows a late bronze age seal from Aegeus with a lion and a horned quadruped [23]. This seal can be viewed as representing a mirror anti-symmetry, because the positions of the animals have a mirror symmetry but animals are inverted with respect to each other, in the sense that one is a predator and the other a prey. In this case, the antisymmtry is not in inverted colours, but in the specific antithetic properties of the subjects.

The hunting and the fighting of wild animals were the favourite themes of Mycenaean population during the Late Bronze Age. Seals with a predator/prey couples were also quite common among Hittites [24]. Seals showing the arrangement of bodies in duels, having bilateral (as in the Figure 7 in the right) and rotational syeemtries, also exist [25]. We could assume the duel as a form of antisymmetry, where a positive hero is facing a negative character.
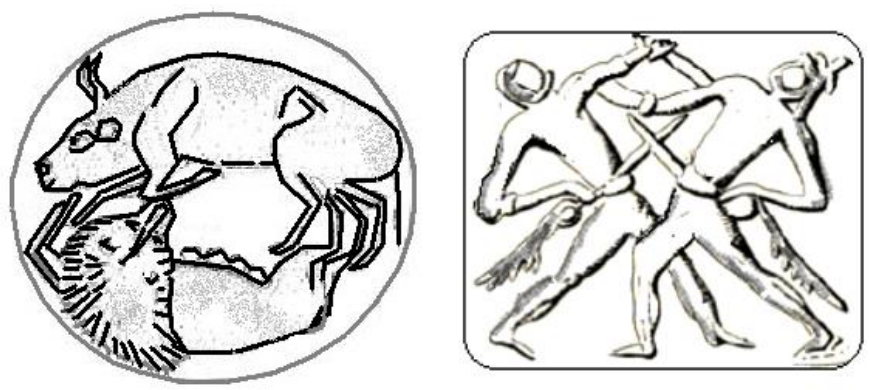

Figure 7. On the left, an image from a late bronze age seal from Aegen area (redrawn from [23]). On the right a duel redrawn fron Ref. 25.

A symmetry can be exact, approximate, or broken. Exact means unconditionally valid, approximate means valid under certain conditions, but broken can mean different things, depending on the considered object and its context. The study of symmetry breaking in physics goes back to Pierre Curie. According to Curie, for the occurrence of a phenomenon in a certain medium, the original symmetry group of the medium must be lowered, that is broken, to the symmetry group of the phenomenon, by means of the action of some cause. Therefore, it is symmetry breaking which creates the phenomenon [26].
The breaking of a certain symmetry does not imply that no symmetry is present, but rather that the situation where this symmetry is broken is characterized by a lower symmetry than the situation where this symmetry is not broken. Symmetry-breaking is a term used in the study of natural languages too: let me consider a phrase from a researcher in linguistic, who tells that "movement is a symmetry-breaking phenomenon" [27], and observe a beautiful Minoan stamp (Figure 8), with men fighting with bulls. This seal has four objects, and then, in principle, it could have a four-fold rotational 
symmetry: the fact that we have two bulls and two men reduces the symmetry to a two-fold one. Moreover, this symmetry is approximate. Note the idea of the motion that this image is able to convey. In the symbolic language of seals, breaking the symmetry means an increase of movement.

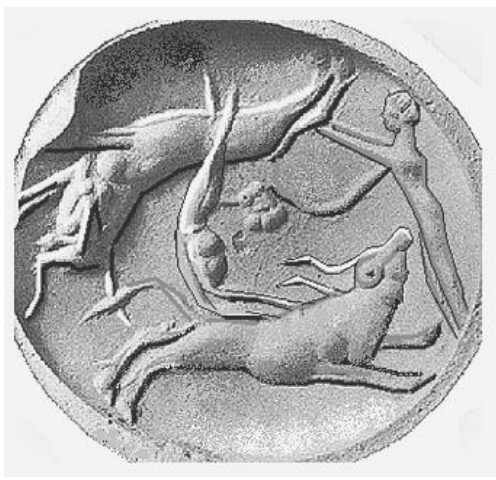

Figure 8. Men fighting with bulls, for Minoan area (redrawn from [28]).

Let us conclude this section noting that during the Late Bronze Age, Mycenaean population adopted the sealing system of Minoan Crete. The most common shape of these seals was the lentoid, with a rich

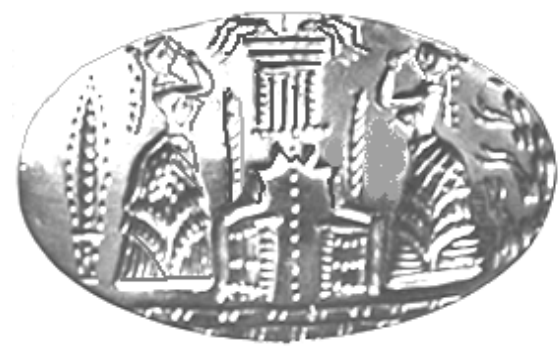

iconography. As the round shape of the seal seems to favour images with rotational symmetries, in the case of lentoid seals, images often display the bilateral symmetry (Figure 9).

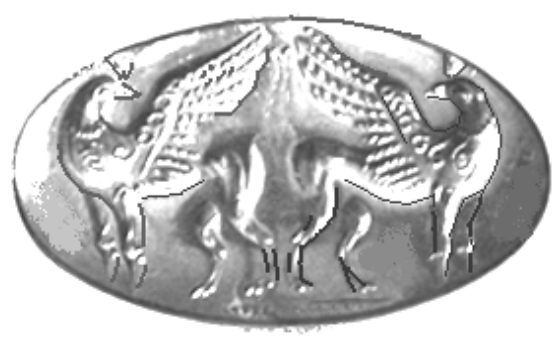

Figure 9. Mycenaean golden rings (redrawn), which were used like seals with bilateral symmetry.

\section{Egyptian scarab seals}

In the case of Egyptian seals we are in the lucky position that many catalogues of huge collections, for instance of the British Museum or of the Egyptian Museum of Cairo [16,17], are in the public domain. As a consequence, we can create a sort of statistics of images and check if symmetries are present and what are the preferred image arrangements. We find a relevant number of stamp seals with cords and coils, highly symmetric, mainly with bilateral symmetry, sometimes with two-fold rotational symmetry. Since scarabs have an elliptic basis, four-fold symmetry is not observed.
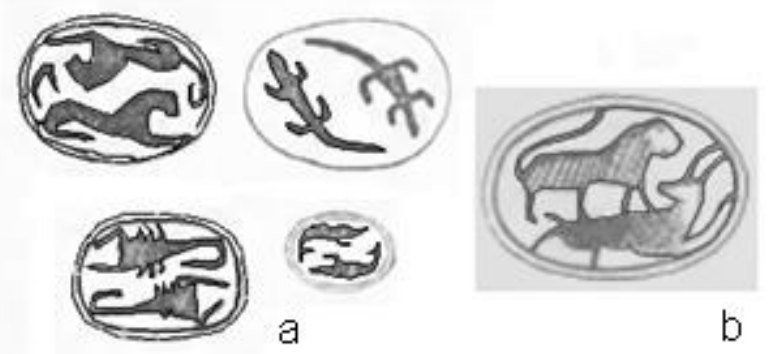

The two-fold symmetry is not frequent among the Egyptian scarab seals, which are depicting animals. In catalogues, we find a few scarabs with two symmetric animals (scorpions, lions, crocodiles or lizards, see Figure 10). It is not clear if these seals were unusual or not enough interesting to deserve a place in a museum collection, because the quality of image is rather poor. Since Scarabs were also amulets, we could guess for those seals having a rotational symmetry, an intent to increase the protection to their owners: the amulet protects in both cases, when it is upright or it is turned upside down.

Figure 10. Images (a) with two-fold rotation symmetry with lions, scorpions and lizards, adapted from [17]. A seal (b) with lion and antelope, adapted from [16], and two seals (c) of the Egyptian Museum Collection, Torino, with bilateral and rotational symmetries. 
The collection of the Egyptian Museum of Torino has a few scarabs with rotational symmetry. We can see a seal with two scorpions and one with two crocodiles. The collection possesses a quite unusual seal with two men, may be twins (Figure 11). This is a cowroid seal, with lentoid shape, imitating the form of a cowry- shell .It is dated to 2200-2040 BCE, the first intermediate period of Egypt. The former scarabs with human figures were developed during this period, depicted in a linear style, as if it were composed by sticks.

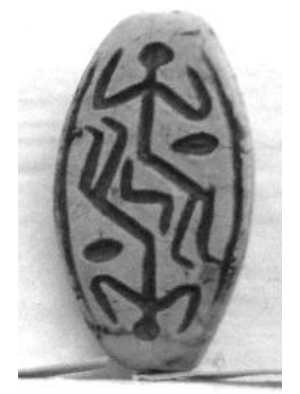

Figure 11. The twins at the Turin Egyptian Museum.

Three-fold, four-fold or greater order rotational symmetries are not observed on scarab seals, because it is difficult to arrange the icons to have these symmetris in an elliptic shape. The collection of the Egyptian Museum of Turin has a seal with a five-fold rosette, but this is a button seals with a round shape. For what concerns the Egyptian knowledge of symmetry, B. Grünbaum claims that Egyptians appear to have missed the three-fold rotation [29]. May be, this symmetry is not used in plane pattern decorations or for scarab seals, but the decoration of Egyptian faience pottery shows a beautiful example of a three-fold symmetry [30].

\section{Conclusion}

This paper discussed the presence of symmetries in images engraved on ancient seals. Bilateral and twofold rotational symmetry are quite common, due to the fact that the base of the stamp seals was often elliptic. Four-fold symmetries are sometimes observed in round stamp seals.

The paper does not pretend to be exhaustive about all the possibilities that can be observed on seals, because the research field and the related literature is huge. This paper would like to be a proposal for a new approach to sillographic analysis, in particular to stimulate the investigations on the meaning of antisymmetries and broken symmetries.

\section{References}

1. G.A. Horridge, The honeybee detects bilateral symmetry and discriminates its axis, Journal of Insect Physiology, Vol.42, 1996, pages $755-764$.

2. J.P. Swaddle and I.C. Cuthill, Preference for symmetric males by female zebra finches, Nature, Vol.367, 1994, pages 165-166.

3. H.B. Barlow and B.C. Reeves, The versatility and absolute efficiency of detecting mirror symmetry in random dot displays, Vision Research, Vol.19, 1979, pages 783-793.

4. J. Wagemans, Detection of visual symmetries, Spatial Vision, Vol.9, 1995, pages 9-32.

5. S. Mancini, S.L. Sally and R. Gurnsey, Detection of symmetry and anti-symmetry, Vision Research, Vol.45, Issue 16, 2005, pages 2145-2160

6. G. Darvas, The interpretation of the concept of symmetry in everyday life, science and art. Symmetry, invariance, harmony, Birkhäuser, Basel, 2007.

7. A.C. Sparavigna, Symmetries in images on ancient seals, Cogprints, November 2008.

8. R. Feynman, R. Leighton and M. Sands, The Feynman Lectures on Physics. 3 volumes 1964,1966.

9. K Brading and E. Castellani, Symmetries in Physics: Philosophical reflections, Cambridge University Press, 2003.

10. D.J. Melville, Aspects of Symmetry in Arpachiyah Pottery, Proceedings of Renaissance Banff: Mathematics, Music, Art. Culture, R. Sarhangi and R.V. Moody (eds.) Renaissance Banff Conference 2005, pages 131-136.

11. Encyclopaedia Britannica, at www.britannica.com w.mdpi.com/journal/algorithms/instructions.

12. A. Türkcan, Stamp Seals, Çatalhöyük Archive report, 1997, available at the web page http://www.catalhoyuk.com/archive_reports/1997/ar97_18.html

13. W. Heimpel, Observations on the meaning and use of preHispanic Mexican stamps, Texas Notes, Vol.60, 1964, pages 1-4.

14. F. Magnarini, Catalogo ragionato di una collezione di scarabeisigillo egizi, BAR International Series 1241, Oxford, 2004.

15. A.C. Sparavigna, Ancient Egyptian seals and scarabs, LULU ebook, 2011.

16. J. Ward, The sacred beetle: a popular treatise on Egyptian scarabs in art and history, John Murray, London, 1902.

17. K.H. Hall, Catalogue of Egyptian scarabs in the British Museum, Royal scarabs, British Museum, London, 1913.

18. P.E. Newberry, Scarab-shaped seals, Archibald Constable and Co., London, 1907.

19. M. Schøyen, Seals, at www.schoyencollection.com/index.html 20. Stamp seals, at www.hindunet.org/hindu_history/sarasvati/lapis/lapis_lazuli.htm

21. P.P.J. Gerdes, On ethno-mathematical research and symmetry. Symmetry in a kaleidoscope, Symmetry Cult. Sci., Vol.1, Issue 2, 1990, pages 154-170.

22. P. Gerdes, Fivefold symmetry and (basket) weaving in various cultures, in I. Hargittai Editor, Fivefold Symmetry, World Scientific, Singapore, 1992

23. Late bronze age stamp seal, at en.wikipedia.org/wiki/Image:Late_bronze_age_seal.jpg

24. C.A. Burney, Historical dictionary of the Hittites, Scarecrow Press, 2004

25. S. O'Brien, S. The role of the duel in early Mycenaean society, www..wac6.org/

26. A discussion on broken symmetries can be found in the Stanford Encyclopaedia of Philosophy, at the link http://plato.stanford.edu/entries/symmetry-breaking/

26. A. Moro, Dynamic antisymmetry: movement as a symmetrybreaking phenomenon, Studia Linguistica, Vol.51, Issue 1, 1997, pages 50-76.

27. S. Davies, Minoan Seal Depicting Bull-Jumping in Action, at web 
www.utexas.edu/courses/introtogreece/lect2/img6minseal.html

29. B. Grünbaum, The emperor's new clothes: full regalia, G-string, or nothing?, Math. Intelligencer, Vol.6, Issue 4, 1984, pages 47-53.

30. A three-fold rotational symmetry is shown by the decoration with fishes and lotus flower of a faience bowl at the Ägyptisches Museum und Papyrussammlung, Berlin. 